Looser, D. (2017). Ausgrenzung, Klassenzusammenhalt, Kooperation und

Selbstbehauptung. Eine quasi-experimentelle Untersuchung der personal-sozialen Kompetenzen bei Schüler/-innen mit einer erlebnispädagogischen Intervention. Gruppe Interaktion Organisation: Zeitschrift für Angewandte Organisationspsychologie, 48(1), 53-60. http://doi.org/10.18747/PHSG-coll3/id/152

Version: This is the accepted version (a post-peer-review, pre-copyedit version) of an article published in Gruppe. Interaktion. Organisation. Zeitschrift für Angewandte Organisationspsychologie (GIO), 48(1), 53-60. The final authenticated version is available online at: http://dx.doi.org/10.1007/s11612-016-0350-6.

Abstract: Mit einem klassischen Quasi-Experimentaldesign (zwei Messpunkte, Behandlungsund Kontrollgruppe) wurden in der 2./3. Schulwoche und nach dem ersten Quartal per Fragebogen die Konstrukte Klassengemeinschaft und Ausgrenzung und Kooperation und Selbstbehauptung erhoben. Die erlebnispädagogische Projektwoche fand in der vierten Schulwoche des neuen Klassenzuges (Schweiz) statt. Die Untersuchung zeigt, dass die Schüler/-innen nach dem Treatment „Projektwoche“ signifikant weniger Ausgrenzungsambitionen und einen besseren Klassenzusammenhalt als die Kontrollgruppen-Schüler/-innen zeigen. Diese Erkenntnis muss unter Einbezug der Störvariablen sinnvoll interpretiert werden. Bezüglich Stichprobe ist zu berücksichtigen, dass die Treatmentgruppe im Vergleich zu den verwendeten Normdaten entweder überdurchschnittlich sozialkompetent war oder einer Stichprobenverzerrung unterlag.

Schlagwörter: Ausgrenzung; Klassenzusammenhalt; Erlebnispädagogik; Soziales Lernen 


\section{Ausgrenzung, Klassenzusammenhalt, Kooperation und Selbstbehauptung. Eine quasi-experimentelle Untersuchung der personal-sozialen Kompetenzen bei Schüler/-innen mit einer erlebnispädagogischen Intervention}

Dölf Looser

\section{Einleitung}

Vor über 200 Jahren zog Wilhelm von Humboldt im Bildungsbericht folgende Bilanz: „Es giebt schlechterdings gewisse Kenntnisse, die allgemein sein müssen, und noch mehr eine gewisse Bildung der Gesinnungen und des Charakters, die keinem fehlen darf" (Humboldt 1809; zitiert nach Flitner und Giel 1982, S. 218). Humboldt ging es demzufolge vor allem um die Charakter- und Gesinnungsbildung, die vor der Berufsausbildung stattfinden sollte. Und heute? Wie wird die „Bildung des Charakters“ von Schüler/-innen umschrieben? Dubs (2010) bezog sich darauf und benutzte den Begriff der Persönlichkeitserziehung, bei der die Bezugspersonen dem Kind/Jugendlichen helfen, die „Grundverhältnisse zu sich selbst (Individuation zum Aufbau der Selbstkompetenz), zu den Menschen (Sozialisation mittels ihrer Sozialkompetenz) sowie zur Welt (Enkulturation mittels der eigenen kulturellen Kompetenz) verantwortungsbewusst und kritisch aufzubauen“ (S. 122). Die angestrebte Vervollkommnung ergebe sich aber nur, wenn es gelingt, ein ausgewogenes Verhältnis zwischen Individuation, Sozialisation und der Enkulturation herbeizuführen, so Dubs.

Der vorliegende Artikel untersucht die Veränderung der vier Konstrukte Klassenzusammenhalt, Ausgrenzung, Kooperation und Selbstbehauptung. Es geht also um Persönlichkeitsbildung und der Förderung von personal-sozialen bzw. sozial-emotionalen Kompetenzen von Schüler/-innen durch soziales Lernen („social emotional learning“ SEL). Im Lernzielkatalog des sozialen Lernens im deutschsprachigen Raum finden sich nach Petillon (2012, S. 107) u. a. die Begriffe Kooperationsfähigkeit, Empathie, Konfliktfähigkeit oder auch Selbstbehauptung. Unter letzterem versteht Petillon, dass man sich für seine Rechte einsetzen lernt und mit Gruppendruck umgehen kann. Während der Gruppendruck im Konstrukt „Ausgrenzung“ (vgl. Instrumente in Abschn. 3.3) ebenfalls eine tragende Rolle spielt, können die beiden Begriffe Selbstbehauptung und Kooperationsfähigkeit in Anlehnung an den neuen „Lehrplan 21“ der Deutschschweiz den zentralen überfachlichen Zielbereichen „personale Kompetenz“ und „soziale Kompetenz“ entsprechend zugeordnet werden. Darum wird in der vorliegenden Studie der Begriff „personal-soziale Kompetenzen“ verwendet. Eine weitergehende Definition der vier zentralen Begriffe findet sich unter 3.3.

Kinder und Jugendliche optimal auf den Beruf und das spätere Leben vorzubereiten, bedeutet, die zentralen überfachlichen Kompetenzen zu fördern. Sie entsprechen den Schlüsselkompetenzen in der heutigen und zukünftigen Gesellschaft (vgl. Gardner 2009). Diese im Sinne Humboldts als „Charakter- und Gesinnungsbildung“ verstandene Bildung hat somit an Bedeutung nichts eingebüßt, gerade weil in unserer Gesellschaft ein enorm großer Wertepluralismus herrscht und der soziale Zusammenhalt nicht wie früher vorausgesetzt werden kann. Es macht darum Sinn, sich einer „erfolgreicheren Vermittlung“ dieser überfachlichen Schlüsselkompetenzen zu widmen, auch wenn sie zumal schwierig nachzuweisen ist. 


\section{Theoretischer Hintergrund}

\section{$2.1 \quad$ Verortung}

Wenn Lehrpersonen von Gewalt-Präventionsprogrammen wie PFADE oder FAUSTLOS als Ansätze zur Förderung überfachlicher Kompetenzen bekannt sind (vgl. auch Malti et al. 2009), bedeutet es jedoch nicht, dass die Persönlichkeitsbildung der Schüler/-innen im Unterrichtsgeschehen als zentraler Aspekt auch behandelt wird. Welche Quellen in Ergänzung zu Dubs (2010) untermauern die Bedeutung der Förderung überfachlicher Kompetenzen bzw. der Persönlichkeitsbildung in der Volksschule? Gemäß Roth (2011) muss der Förderung der Persönlichkeitsbildung der Schüler/-innen eine ebenso große Bedeutung wie der Wissensvermittlung zukommen und sollte einerseits in stärkerem Maße und andererseits professioneller in den Schulen stattfinden (S. 291). Auch Stanford (2010) fordert gerade zu Beginn einer Klassenbildung die explizite Beschäftigung mit der Gruppendynamik und dem Kennenlernen von Regeln in der Klasse - initiiert durch die Klassenlehrperson. In Anlehnung an Böttcher und Lindart (2009) können Vertreter einer Schule, welche verstärkt auf die Entwicklung von überfachlichen Schlüsselqualifikationen achten, sich auf zwei prominente Argumente stützen:

Zunächst ist die Schule selbst ein Ort sozialen Geschehens. (...) Einerseits soll die Gestaltung des schulischen Lebens selbst dazu beitragen, ... damit die Schule zu einem Ort des Miteinander wird und ein positives Lernklima die Leistungen und das Wohlbefinden verbessern hilft. (...) Ein zweites Kernargument für die Vermittlung von Schlüsselqualifikationen findet sich in der wohl selbstverständlichen Philosophie, dass Schule die jungen Menschen „auf das Leben“ vorzubereiten habe. (...) Wer junge Menschen nicht nur „um der Bildung willen“ schult, sondern sie auch ausrüsten will für die beruflich zu bewältigenden Aufgaben, wird keine Not haben, soziale Kompetenzentwicklung als wichtige pädagogische Aufgabe der Schule einzufordern (Böttcher und Lindart 2009, S. 22).

Die genannten Programme PFADE usw. nehmen sich dieser Aufgabe der Schlüsselqualifikationsvermittlung sicherlich an, wobei sie auf Reihen von bis zu 47 Lektionseinheiten basieren, die über das halbe oder ganze Jahr verteilt werden. Das in Kap. 3 vorgestellte Programm unterscheidet sich von solchen Lektionsreihen, in denen die Thematik wöchentlich „unterrichtet“ wird. Die Förderung der überfachlichen Kompetenzen erfolgt über eine kompakte fünftägige Projektwoche „Die Gruppe und ich“, bei der die Schüler/-innen der 1. Oberstufe praktische Erfahrungen und zentrale Erkenntnisse rund um die Gruppendynamik handelnd erfahren und verarbeiten. Diese Intervention lehnt sich an die Grobeinteilung ${ }^{1}$ nach Heyse und Erpenbeck (2004) an, wobei im Sinne der Veränderung des Sozialverhaltens mittels der Methode Selbsterfahrung (Gruppendynamik, Outdoortraining etc.) und der Wissensvermittlung in klassenübergreifenden Gruppen gearbeitet wird.

\subsection{Gruppendynamik und personal-soziale Kompetenzen}

Das Projekt „Die Gruppe und ich“ beinhaltet u. a. die großen Themenschwerpunkte Gruppendynamik, Schulgemeinschaft/Klassengemeinschaft, Ausgrenzung und Selbstbehauptung. Diese Konstrukte werden im Folgenden bezüglich ihres Zusammenhangs mit Gruppendynamikprozessen verortet.

Die Bedeutung der Lehrperson ist bei der Gruppenentwicklung der Klasse alles andere als unwichtig: Ihr Einfluss auf eine möglichst positive Gruppenentwicklung ist gemäß Stanford (2010) vor allem im Stadium „Einführung von Normen“ - und somit vor dem Konfliktstadium im Gruppenentwicklungsprozess - sehr zentral. Die folgende Abb. 1 verweist in der oberen

\footnotetext{
${ }^{1}$ Wissensvermittlung; Verhaltenstraining; Beratung und Selbsterfahrung.
} 
Zeile auf die Stadien der Gruppenentwicklung, welche der Reihe nach durchlebt werden: Orientierungsphase, Einführung von Normen, Konfliktphase, Produktivitätsphase und Auflösungsphase. Die Thematisierung der Gruppennormen sorgt letztlich dafür, dass die Gruppe nach einer möglichst kurzen Konfliktphase, wo kleinere Durchsetzungsprozesse einzelner Gruppenmitglieder stattfinden, in der Phase der Produktivität lange erfolgreich verweilen kann.

Der Umgang mit dem Gruppenentwicklungsprozess im thematisierten Programm unterscheidet sich daher von klassischen Ansätzen, bei denen sich die Lehrperson bzw. die Schule nicht einmischt (z. B. Tuckman 1965; nach König und Schattendorfer 2011, S. 61). Der Übergang von Anpassungsdruck, Gruppendruck und Mobbing ist erfahrungsgemäß häufig schleichend. Wenn es um die Prävention von Ausgrenzung bzw. um die in einer Klasse gelebte Meinungspluralität geht, muss sich die Lehrperson fragen, ob es Cliquen oder Schüler/-innen in einer Klasse gibt, die den Ton angeben bzw. die andere Schüler/-innen in ihrer Meinung unter Druck setzen (vgl. Konstrukt Ausgrenzung bzw. Meinungspluralität unter Abschnitt Erhebungsinstrument).

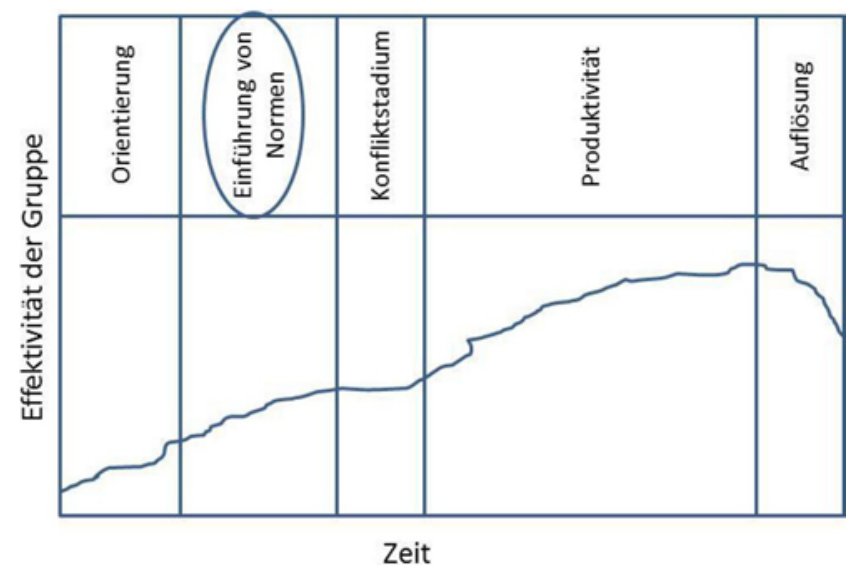

Abb. 1 Gruppenentwicklung und Leistungsfähigkeit nach Stanford (vgl. 2010, S. 18) skizziert durch Autor

Mit der viertägigen Projektwoche wird beabsichtigt, solche oft nicht optimal verlaufenden Gruppenentwicklungsprozesse zu Beginn der Klassenbildung präventiv aufzufangen und in Richtung positiver Schul- und Klassengemeinschaft, Kooperation und Selbstbehauptung zu lenken.

\subsection{Erlebnispädagogische und gruppendynamisch Elemente}

Die Präventionswoche „Die Gruppe und ich“ beinhaltete nebst gruppendynamischen auch erlebnispädagogische Elemente. Erlebnispädagogik kann insofern charakterisiert werden, als durch Erfahrung und Erleben intensiv und nachhaltig gelernt wird (vgl. bspw. Reiners 1997). Gilsdorf (2003) führt als Leitmotive der Erlebnispädagogik u. a. unmittelbare Erfahrung, persönliche Bedeutsamkeit des Tuns, reflektierendes Handeln, Kooperation sowie Prozessorientierung an.

Zur Wirksamkeit von Projektwochen mit gruppendynamischen und erlebnispädagogischen Elementen lässt sich folgendes festhalten: In einer Studie mit 48 11bis 13Jährigen von Moch (2002) zeigten sich nach einer einwöchigen Intervention mit erlebnispädagogischen Maßnahmen positive und zeitlich stabile Veränderungen im eingeschätzten Gruppenzusammenhalt und Selbstvertrauen. Weiterhin zeigten sich in einer Studie von Zick 
(2002) mit 39 Jugendlichen im Alter von 16 und 17 Jahren positive Effekte einer erlebnispädagogischen Intervention auf die Einstellungen zur Schule, Identifikation mit der Schule bzw. Klasse und die Verhaltensintentionen. Boeger et al. (2006) zeigten in einer sechsmonatigen Prä-Post-Kontrollgruppenstudie Verbesserungen im Selbstwertgefühl und der psychosozialen Befindlichkeit durch eine erlebnispädagogische Intervention im schulischen Kontext bei 122 Jugendlichen. Es kann bilanziert werden, dass erlebnispädagogische Interventionen auf die ganzheitliche Entwicklung der Persönlichkeit sowie auf die Förderung individueller Kompetenzen bei der Bewältigung von Alltagsproblemen abzielen (vgl. u. a. Moch 2002).

Nicht nur ist eine Projektwoche mit gruppendynamischen und erlebnispädagogischen Elementen im Sinne der ganzheitlichen Persönlichkeitsentwicklung zur Förderung personalsozialer Kompetenzen sinnvoll und die Normsetzung zu Beginn einer Klassenbildung von zentraler Bedeutung (vgl. Stanford 2010), auch soll sie zur Förderung des Klassenzusammenhalts und zu einem bewussten Umgang mit Gruppendruck und Selbstbehauptung beitragen (vgl. u. a. Steins et al. 2014).

\section{Methode}

\subsection{Forschungsfrage}

Ziel der Studie ist es, mittels eines Quasi-Experimentaldesigns bei Schüler/-innen der 1. Oberstufe die Wirkung einer klassenübergreifenden Projektwoche mit gruppendynamischen und erlebnispädagogischen Elementen in den abhängigen Variablen Klassengemeinschaft, Ausgrenzung, Kooperation und Selbstbehauptung zu untersuchen. Insofern stehen die Interaktionen der „experimentellen Bedingung“ mit „Zeit“ sowie die Wechselwirkung der Faktoren „Geschlecht", „experimentelle Bedingung“ und „Zeit" im Vordergrund des Interesses. Ziel der Intervention ist es, die in der Einleitung beschriebenen zentralen Grundverhältnisse zu sich selbst und zu Mitschüler/-innen und Erwachsenen verantwortungsbewusst aufzubauen, indem gruppendynamische Prozesse und diesbezügliche Schlüsselerkenntnisse über aktive Beschäftigung und Wissensvermittlung in der klassenübergreifenden erlebnispädagogischen Projektwoche bewusst erarbeitet werden. ${ }^{2}$ Eine mitunter so initiierte neue Einstellung zu Klassen-/Schulnormen und zur selbst mitgestalteten Gruppenentwicklung in der Klassengemeinschaft anfangs Schuljahr ist im Sinne der Sozialisation beabsichtigt und macht insbesondere auf der in der Schweiz mit dem 7. Schuljahr beginnenden neuen Oberstufenschulzeit Sinn, weil die Gruppe in der Adoleszenz eine besondere Bedeutung erhält und die Klassen neu zusammengesetzt sind.

\subsection{Stichprobe und Durchführung}

An der Untersuchung haben insgesamt 13 Oberstufenschulklassen $(n=226)$ teilgenommen. Dabei sind mit einem quasi-experimentellen Design (zwei Messpunkte, Behandlungs- und Kontrollgruppe) in der 2./3. Schulwoche und nach dem ersten Quartal per Fragebogen die Konstrukte Klassengemeinschaft und Ausgrenzung (vgl. Bundeszentrale für politische Bildung 2012) und Kooperation und Selbstbehauptung (vgl. Petermann und Petermann 2013) erhoben worden. Die Projektwoche mit gruppendynamischen und erlebnispädagogischen Elementen hat in der vierten Schulwoche stattgefunden.

In dieser nicht-randomisierten Studie wird ein dreifaktorielles Design (experimentelle Bedingung, Geschlecht, Messzeitpunkt) einerseits und die Testung mit normierten Populationsdaten andererseits vorgenommen. Die Intervention dieser Projektwoche beinhaltete Problemlöse-, Vertrauens- und Kooperations-, Interaktionsübungen,

\footnotetext{
2 Die Zielsetzung der Woche wurde den Schüler/-innen explizit zu Beginn der Projektwoche
} kommuniziert. 
erlebnispädagogische Aktivitäten (z. B. Wettbewerbsspiele abends im Wald) sowie regelmäßige Reflexionen zur Förderung sozialer Kompetenzen und der Teamarbeit als auch die Beschäftigung mit dem Film „Herr der Fliegen“. Die Kontrollgruppe war in Bezug auf das Alter, die Schulstufe und den Schultyp parallelisiert.

Von den sechs Klassen der Experimentalgruppe und den sieben Klassen der Kontrollgruppe mussten bei der Analyse vier Klassen ausgeschlossen werden. Zum einen sind in der Experimental- und Kontrollgruppe Mobbingfälle aufgetreten, welche durch die Schulsozialarbeiter/innen genau zwischen der t1und t2-Befragung bearbeitet wurden. Zum anderen haben Kontrollklassenlehrpersonen mit ihren Schüler/-innen in dieser Phase besonders im Bereich soziales Lernen gearbeitet, was einer Intervention ähnlich kam und so den Kontrollcharakter erübrigte. Aufgrund der Analyse dieser Klassen und dem Ausschluss derer Daten wurde die Kontroll- und Experimentalgruppe schließlich bereinigt, was sich letztlich auf Kosten der Stichprobengröße auswirkte - statt insgesamt 212 Probanden konnten für die Berechnungen nur noch 129 Schüler/-innen (84 Experimental- und 45Kontrollgruppe) miteinbezogen werden. Da die Suche nach Kontrollgruppen schwierig war und der Forschungsansatz einen quasi-experimentellen und einen nicht randomisierten Ansatz verfolgt, ist der Stichprobenreduktion nicht erfreulich, aber forschungstechnisch nachvollziehbar.

Die erlebnispädagogisch und gruppendynamisch orientierte Projektwoche „Die Gruppe und ich" wurde von Montag bis Freitag umgesetzt, wobei die Woche vor der Intervention die Erhebung $\mathrm{t} 1$ und sieben Wochen danach die Erhebung t2 durchgeführt wurde. Die erlebnispädagogischen Übungen boten den Jugendlichen Problemlösungsprozesse, welche nach einem Phasenmodell (vgl. Gilsdorf und Volkert 1999) strukturiert waren: Es zeigt sukzessive aufsteigend die Stufen des Problemlösungsprozesses von der anfänglichen Erfassung der Problemstellung über die Beratung in der Gruppe über mögliche Lösungsstrategien, die praktische Phase der Umsetzung der entwickelten Strategie bis hin zur Bewertung des Ergebnisses und zur Rückschau auf die Besonderheiten im Verlauf der Übung. Dieses Phasenmodell wurde sowohl in den einzelnen Modulen als auch über die gesamte Woche hinweg benutzt: Aktion und Reflexion waren stets aufeinander bezogen. Zudem wurde die Projektwoche im nachfolgenden Quartal als Ankererfahrung bei weiteren Übungen zum sozialen Lernen wieder thematisiert, um einen möglichst nachhaltigen Effekt der Projektwoche auf den Klassenzusammenhalt und die personal-sozialen Kompetenzen zu erreichen.

\subsection{Instrumente}

Die Projektwoche gründet auf der grundsätzlichen Annahme, die subjektive Einschätzung der beiden Skalen,

Klassenzusammenhalt und Ausgrenzung, mittels Projektwoche bei den Schüler/-innen positiv zu verändern. Beide Konstrukte (vgl. Bundeszentrale für politische Bildung (bpb) 2012) weisen Reliabilitätswerte (Cronbachs Alpha) von 0,73 und 0,68 auf, bei Klassenzusammenhalt mit drei Items, bei Ausgrenzung mit fünf. Während der Klassenzusammenhalt durch Items wie „Wir haben eine gute Klassengemeinschaft“ oder „In meiner Klasse habe ich mehrere gute Freunde oder Freundinnen" repräsentiert wird, geht es beim Konstrukt Ausgrenzung bzw. Meinungspluralität um Aspekte wie „Bei uns werden einzelne Schüler/innen ausgeschlossen“ oder „In unserer Klasse gibt es Cliquen oder Schüler/innen, die den Ton angeben“. Besonders das Item „Bei uns wird man schnell zum Aussenseiter, wenn man nicht tut oder sagt, was die anderen wollen" weist auf den gruppendynamischen Aspekt der Ausgrenzung bzw. Meinungspluralität hin und stimmt insofern überein mit dem Projektwochentitel „Die Gruppe und ich“, wo ein sinnvoller Umgang mit Gruppendruck und Gruppendynamik thematisiert wird. 
Die zwei personal-sozialen Kompetenzbereiche, welche im Rahmen der Projektwoche ebenfalls untersucht wurden, sind der Schülereinschätzliste für Sozial- und Lernverhalten (SSL) nach Petermann und Petermann (2013) entnommen. Die internen Konsistenzen der Messbereiche des SSL liegen zwischen 0,82 und 0,95 (Cronbachs Alpha). Es handelt sich um sehr homogene Aussagebereiche. Die zwei Kompetenzbereiche lassen sich wie folgt beschreiben (vgl. ebd.):

Kooperation erfasst das Ausmass, in dem ein Schüler mit Mitschülern zusammenarbeitet und am gemeinsamen Lernen teilnimmt. Es geht um die Fähigkeit zum kooperativen Lernen, wo z. B. zuhören, Rückmeldungen einholen und geben und das Einbeziehen von anderen Schüler/-innen gefragt ist. Angemessene Selbstbehauptung erfasst das Ausmass, in dem ein Schüler in konfliktreichen Situationen angemessen reagiert und Streit mit anderen Mitschülern friedlich lösen kann. Da das Copyright der SSL-Skalen eingehalten werden muss, wird auf die Nennung von Beispielitems verzichtet.

\section{Ergebnisse}

Anhand von Varianzanalysen mit Messwiederholung werden der Reihe nach die Wirkung der Präventionswoche auf die abhängigen Variablen Klassenzusammenhalt, Ausgrenzung, Kooperation und Selbstbehauptung untersucht.

\subsection{Klassenzusammenhalt}

Die Varianzanalyse mit Messwiederholung mit dem Faktor Zeit (t1/t2) und den beiden Faktoren Gruppe (Experimentalgruppe/Kontrollgruppe) und Geschlecht (weiblich/männlich) zeigt bezüglich der abhängigen Variablen Klassenzusammenhalt mit den Kennzahlen ein signifikantes Resultat in Bezug zur Interaktion von Gruppe x Zeit $(F(1,125)=9,682 ; p<0,05$; $\eta 2=0,072$ ), aber nicht in Bezug zur Interaktion Zeit * Gruppe * Geschlecht. Der signifikante Interaktionseffekt Zeit * Gruppe weist ein Eta-Quadrat von $\eta 2=0,072^{3}$ aus. Insofern kann die beabsichtigte Wirkung der Intervention bei der Kontrollgruppe mit einem großen Effekt nachgewiesen werden (vgl. Abb. 2). Der dreifache Interaktionseffekt Zeit * Geschlecht * Zeit zeigt sich nicht signifikant, d. h. der Klassenzusammenhalt ist vom Geschlecht nicht zusätzlich abhängig.

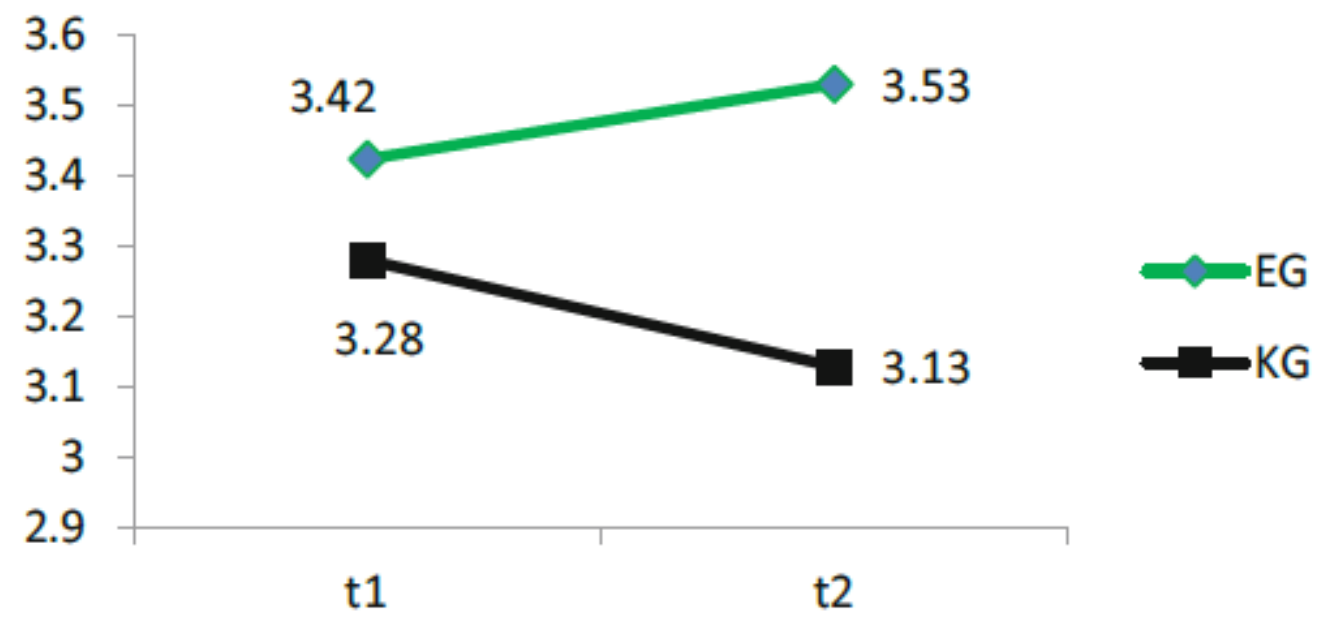

\footnotetext{
${ }^{3}$ Gemäß Rost (2005) kann bei einem Eta-Quadrat n2 ab 0,01 von einem kleinen Effekt, ab 0,06 von einem mittleren Effekt und ab 0,25 von einem großen Effekt gesprochen werden.
} 
Abb. 2 Klassenzusammenhalt

\subsection{Ausgrenzung}

Bei der Überprüfung der Ausgrenzung innerhalb der einzelnen Schulklassen zeigt sich ebenfalls die beabsichtigte Wirkung, jedoch mit einer kleineren Effektstärke. Mittels der gleich gesetzten Varianzanalyse mit Messwiederholung zeigen sich die einzelnen Interaktionseffekte Zeit * Gruppe $(F(1,125)=6,717 ; p<0,05 ; \eta 2=0,051)$ und Zeit * Geschlecht $(F(1,125)=7,242 ; p<0,05 ; \eta 2=0,055)$ signifikant. Es kann also bilanziert werden, dass die Experimentalgruppe im Vergleich zur Kontrollgruppe signifikant tiefere Ausgrenzungsambitionen nach der Präventionswoche zeigt. Dabei sind es vor allem Mädchen in den Kontrollklassen, welche im zweiten Messzeitpunkt relativ hohe Ausgrenzungstendenzen in ihrer Klasse wahrnehmen (vgl. Abb. 3).

\subsection{Kooperation und Selbstbehauptung}

Bei den Skalen Kooperation und Selbstbehauptung kann auf Populationswerte (Petermann und Petermann 2013) zurückgegriffen werden. Gemäß Abb. 4 fällt bei der Skala Kooperation auf, dass die Experimentalgruppe schon zu Beginn (t1) ein relativ hohes Niveau zeigt - ganz im Gegensatz zur Kontrollgruppe. Dies ist in einem Quasi-Experiment (wo das Treatment in den einzelnen Schulklassen nicht randomisiert werden konnte) nachvollziehbar, jedoch nicht ganz wunschgemäß.
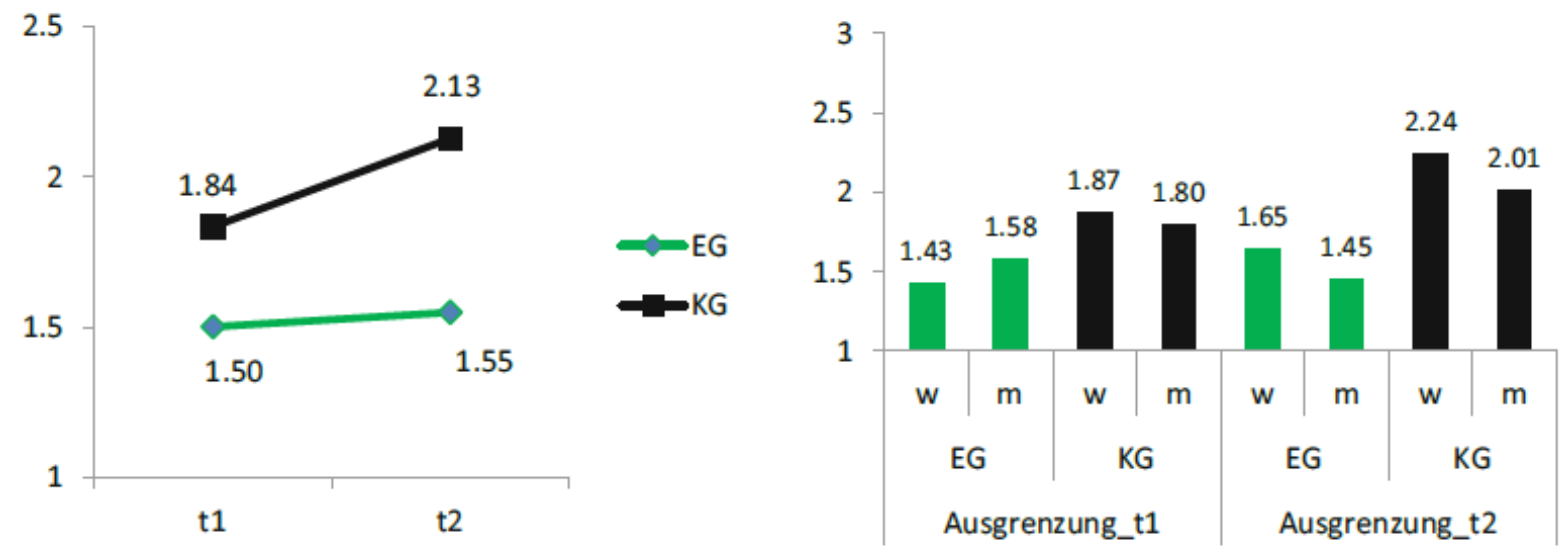

Abb. 3 Ausgrenzung total (a) und Ausgrenzung geschlechtsspezifisch (b)
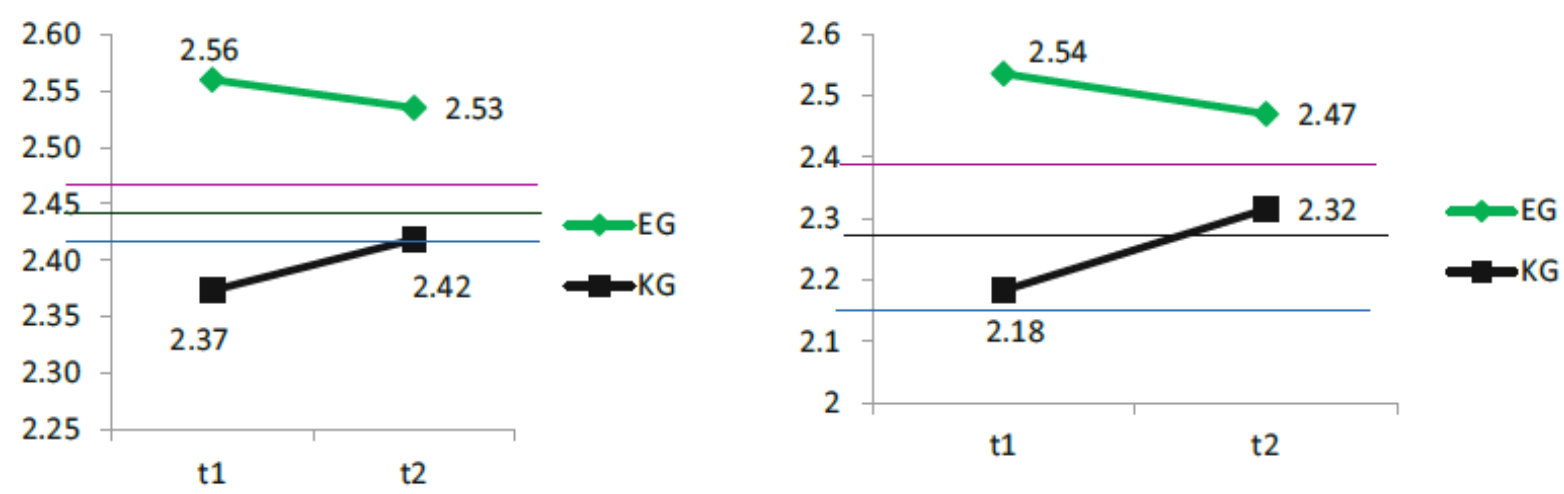

Abb. 4 Kooperation (a) und Selbstbehauptung (b) 
Anhand der Kennwerte $(F(1,126)=0,874 ; p=0,35 ; \eta 2=0,007 ; n$. s. $)$ kann kein signifikanter Effekt Zeit * Gruppe nachgewiesen werden. Auch erweisen sich die Interaktionen Zeit * Geschlecht und Zeit * Gruppe * Geschlecht als nicht signifikant. Im Vergleich zur Normstichprobe (vgl. Abb. 4 - untere Linie für männliche Jugendliche und obere Linie für weibliche Jugendliche) bleibt die Experimentalgruppe auf überdurchschnittlichem Niveau in t1 und t2, wohingegen die Kontrollgruppe zum Zeitpunkt t2 zum durchschnittlichen Niveau aufschliesst. Die mittlere Linie zeigt den geschlechtsunabhängigen Normwert der Skala „Kooperation“ in Deutschland bei einer Skala von 0 bis 3.

Das Konstrukt „Selbstbehauptung“ wurde ebenfalls mittels einer Varianzanalyse mit Messwiederholung untersucht. Auch hier kann wieder dieselbe Verzerrung beobachtet werden (vgl. Abb. 4): Die Experimentalgruppe weist einen überdurchschnittlichen Wert in Selbstbehauptung auf, während die Kontrollgruppe sich im Normbereich aufhält. Insofern ist es wenig erstaunlich, dass der Interaktionseffekt Zeit * Gruppe in umgekehrter Richtung als erwartet ausfällt $(F(1,126)=9,179 ; p<0,05 ; \eta 2=0,068)$. Es kann von einem Ceiling-Effekt gesprochen werden (der maximale Wert in der Skala liegt bei 3).

Die Ergebnisse weisen insgesamt gesehen eine positive Bilanz der Projektwoche mit gruppendynamischen und erlebnispädagogischen Elementen aus (vgl. Tab. 1).

\begin{tabular}{|c|c|c|c|c|c|c|c|c|c|}
\hline & $\begin{array}{l}\text { Ausgrenzung } \\
\mathrm{t} 1\end{array}$ & $\begin{array}{l}\text { Ausgrenzung } \\
\text { t2 }\end{array}$ & $\begin{array}{l}\text { Klassen- } \\
\text { zusammen- } \\
\text { halt t1 }\end{array}$ & $\begin{array}{l}\text { Klassen- } \\
\text { zusammen- } \\
\text { halt t2 }\end{array}$ & \multicolumn{2}{|c|}{$\begin{array}{l}\text { Kooperation } \\
\mathrm{t} 1\end{array}$} & $\begin{array}{l}\text { Kooperation } \\
\text { t2 }\end{array}$ & $\begin{array}{l}\text { Selbst- } \\
\text { behauptung } \\
\mathrm{t} 1\end{array}$ & $\begin{array}{l}\text { Selbst- } \\
\text { behauptung } \\
\mathrm{t} 1\end{array}$ \\
\hline $\begin{array}{l}\mathrm{sd} \\
\mathrm{n}\end{array}$ & \begin{tabular}{|rr|} 
EG & \multicolumn{1}{l|}{ KG } \\
0.42 & 0.46 \\
84 & 45 \\
\end{tabular} & 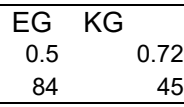 & \begin{tabular}{|rr} 
EG & KG \\
0.49 & 0.53 \\
84 & 44 \\
\end{tabular} & \begin{tabular}{|cc} 
EG & EG \\
0.47 & 0.6 \\
84 & 84 \\
\end{tabular} & $\begin{array}{r}\text { EG } \\
0.44 \\
84 \\
\end{array}$ & $\begin{array}{r}\mathrm{KG} \\
0.45 \\
46 \\
\end{array}$ & \begin{tabular}{|rr} 
EG & EG \\
0.44 & 0.47 \\
84 & 46 \\
\end{tabular} & \begin{tabular}{|rr|} 
KG & \multicolumn{1}{l|}{ EG } \\
0.53 & 0.53 \\
46 & 84 \\
\end{tabular} & \begin{tabular}{|rr|} 
KG & \multicolumn{1}{l|}{ KG } \\
0.45 & 0.64 \\
46 & 44 \\
\end{tabular} \\
\hline $\begin{array}{l}\text { Zeit * } \\
\text { Gruppe }\end{array}$ & \multicolumn{2}{|c|}{$\begin{array}{l}F_{(1,125)}=6.717 ; p<.05 \\
\eta 2=.051\end{array}$} & \multicolumn{2}{|c|}{$\begin{array}{l}F_{(1,125)}=9.682 ; p<.05 \\
\text { १2=.072 }\end{array}$} & \multicolumn{3}{|c|}{$\begin{array}{l}F_{(1,126)}=0.874 ; p=.35 \\
\text { n2=.007; n.s. }\end{array}$} & \multicolumn{2}{|c|}{$\begin{array}{l}F(1,126)=9.179 ; p<.05 \\
\eta 2=.068\end{array}$} \\
\hline Bilanz & \multicolumn{2}{|l|}{ These bestätigt } & \multicolumn{2}{|c|}{ These bestätigt } & \multicolumn{3}{|c|}{$\begin{array}{l}\text { These nicht bestätigt, } \\
\text { Ausgangsniveau bei KG zu } \\
\text { hoch }\end{array}$} & \multicolumn{2}{|c|}{$\begin{array}{l}\text { These nicht bestätigt, } \\
\text { Ausgangsniveau bei KG zu } \\
\text { hoch }\end{array}$} \\
\hline
\end{tabular}

Tab. 1 Zusammenfassung der Wirkungen

Die Schüler/-innen nehmen aufgrund der Intervention im Zeitpunkt t2 den Klassenzusammenhalt signifikant positiver wahr und zeigen sich in ihren Ausgrenzungsambitionen signifikant tiefer als die Kontrollschüler/-innen in den jeweiligen Klassen. Die signifikanten Interaktionseffekte können als mittelstark (Rost 2005, S. 173) bezeichnet werden. Im Gegensatz dazu können aber bei den SSL-Skalen Kooperation und Selbstbehauptung die beabsichtigen Wirkungen nicht nachgewiesen werden. Es wird auf den aus der quasi-experimentellen Anlage entstandenen Stichprobenfehler verwiesen: Die Experimentalgruppe weist schon zu Beginn (t1) signifikant höhere Werte in Kooperation und Selbstbehauptung als die deutsche Normstichprobe auf. Die Kontrollstichprobe ihrerseits unterscheidet sich aber zu Beginn (t1) nicht signifikant von der Norm.

\section{Diskussion}

Die Untersuchung zeigt, dass die Schüler/-innen, welche die erlebnispädagogische Intervention „Projektwoche“ erfahren haben, signifikant weniger Ausgrenzungsambitionen als die Kontrollgruppen-Schüler/-innen zeigen. Da die Erhebung mit im Vergleich zu den Normdaten aus Deutschland (Petermann und Petermann 2013) überdurchschnittlich 
sozialkompetenten Schüler/-innen in der Treatmentgruppe stattgefunden hat, die Kontrollstichprobe aber durchschnittlich war, zeigen sich die Wirkungseffekte des Treatments bei zwei Skalen nur moderat bzw. bei zwei Skalen gar nicht. Dennoch muss generell bedacht werden, dass hierbei Stichprobenverzerrungen mitspielen können und eine Zeitspanne zwischen $\mathrm{t} 1 \mathrm{und} \mathrm{t} 2$ von acht Wochen nicht überinterpretiert werden darf, wenn es um Konstrukte der Persönlichkeitsbildung geht. Dabei können etliche Störvariablen (Familie, schulische Prozesse, ...) ebenfalls einen Einfluss gehabt haben. Aufgrund der theoriekonformen Teil-Ergebnisse dürfte es sich lohnen, die Überprüfung der Wirkungen mit weiteren Stichproben - ohne Ceiling-Effekt bzw. ohne mögliche Stichprobenverzerrung - in einer erweiterten Untersuchungsanlage vorzunehmen. Denn auch das Bilanzitem „Durch die Projektwoche habe ich vieles über den Umgang in einer Gruppe gelernt" wird von den betroffenen Schüler/-innen zu $88 \%(n=84)$ positiv bewertet und die Schüler-Antworten auf die offene Frage weisen in eine ähnliche Richtung hin. Bei quasi-experimentellen Studien muss die Begrenztheit der Interpretation von beabsichtigten Wirkungen immer bedacht werden: Es lassen sich nie alle Störvariablen kontrollieren, insofern können auch unbeabsichtigte Wirkungsmechanismen nicht ausgeschlossen werden. Die hier gelieferten Erkenntnisse sollen die praktizierenden Lehrpersonen motivieren, sich den zwar schwierig zu messenden aber sehr bedeutsamen Konstrukte der personal-sozialen Kompetenzen anzunehmen und diese (gemäß Schweizer Lehrplan 21) bestmöglichst zu fördern, auch wenn sich dabei nicht alle Nebenwirkungen ausschliessen lassen.

Auf diesen Punkt bezogen könnte man in Anlehnung an Petillon (2012) erwarten, dass „positives Sozialverhalten in der Familie“ gelehrt wird. Aufgrund „entwicklungsstörender Einflüsse und familiärer Erziehungsprobleme" seien diese primären Prozesse des sozialen Lernens aber „immer häufiger beeinträchtigt“ (S. 107), so Petillon. Daraus könnte die Forderung abgeleitet werden, dass soziales Lernen in der Schule systematischer gelehrt werden sollte. Der entsprechende Lernzielkatalog des sozialen Lernens, auf den Bezug genommen wurde, wurde in der Einleitung erwähnt.

Diese Studie hat sich sowohl der Zielsetzung nach der systematischen Förderung des sozialen Lernens als auch der Förderung des Klassenzusammenhalts und der Entwicklung personal-sozialer Kompetenzen angenommen. Eine gesteuerte Gruppenentwicklung kann nicht nur zur Förderung der Klassengemeinschaft beitragen (Stanford 2010, S. 20 ff), sondern auch eine größere Schulzufriedenheit bei den Schüler/-innen bewirken - weil Schule in der Wahrnehmung so gerechter und sicherer wird (Samdal et al. 1998). Zudem ist auch von positiven Effekten in Bezug zu einer funktionierenden Klassenführung und von guten Beziehungen auszugehen. Gute soziale Beziehungen, sowohl zwischen Schüler/innen als auch zwischen Schüler/-innen und Bezugspersonen, sind letztlich für die erfolgreiche Lebensbewältigung von zentraler Bedeutung, wie dies Längsschnittuntersuchungen eindrücklich gezeigt haben (Fend 1991; Looser 2011). So verstanden wurde versucht, der Humbolt'schen Charakter- und Gesinnungsbildung in begrenztem Umfang Rechnung zu tragen.

\section{Literatur}

Boeger, A., Dörfler, T., \& Schut-Ansteeg, T. (2006). Erlebnispädagogik mit Jugendlichen Einflüsse auf Symptombelastung und Selbstwert. Praxis der Kinderpsychologie und Kinderpsychiatrie, 55, 181-197.

Böttcher, W., \& Lindart, M. (2009). Schlüsselqualifiziert: Schüler entwickeln personale und soziale Kompetenzen. Weinheim: Beltz. Bundeszentrale für politische Bildung (bpb) (2012). Projekt Klassen-CheckUp! Bonn. http://www.bpb.de/lernen/. Zugegriffen: 22. Januar 2014. 
Dubs, R. (2010). Bildungspolitik und Schule - wohin? Altstätten: Verlag Tobler.

Fend, H. (1991). Schule und Persönlichkeit: Eine Bilanz der Konstanzer Forschungen zur "Sozialisation in Bildungsinstitutionen“. In R. Pekrun \& H. Fend (Hrsg.), Schule und Persönlichkeitsentwicklung (S. 9-32). Stuttgart: Enke.

Flitner, A., \& Giel, K. (Hrsg.). (1982). Schriften zur Politik und zum Bildungswesen. Wilhelm von Humboldt - Werke in fünf Bänden, Bd. IV. Darmstadt: Wissenschaftliche Buchgesellschaft.

Gardner, H. (2009). Five Minds for the Future. Cambrigde: Harvard Business Review Press.

Gilsdorf, R. (2003). Die Konzepte „Abenteuer“ und „Projekt“ - Über die Prinzipien erfahrungsorientierten Lernens. Schulmagazin, 2, 5-10.

Gilsdorf, R., \& Volkert, K. (1999). Abenteuer Schule. Alling: Sandmann.

Heyse, V., \& Erpenbeck, J. (2004). Kompetenztraining. Stuttgart: Schäffer-Poeschel.

König, O., \& Schattenhofer, K. (2011). Einführung in die Gruppendynamik. Heidelberg: Carl-Auer.

Looser, D. (2011). Soziale Beziehungen und Leistungsmotivation. Die Bedeutung von Bezugspersonen für die längerfristige Aufrechterhaltung der Lernund Leistungsmotivation. Opladen \& Farmington Hills MI: Budrich Unipress.

Malti, T., Häcker, T., \& Nakamura, Y. (2009). Kluge Gefühle? Sozial-emotionales Lernen in der Schule. Zürich: Verlag Pestalozzianum.

Moch, M. (2002). Entwicklung von Gruppenstruktur, Zusammenhalt und Selbstvertrauen im Verlauf erlebnispädagogischer Segelmaßnahmen. Gruppendynamik und Organisationsberatung, 33, 83-95.

Petermann, U., \& Petermann, F. (2013). Schülereinschätzliste für Sozial- und Lernverhalten (SSL). Göttingen: Hogrefe.

Petillon, H. (2012). Soziales Lernen. In G. Keller (Hrsg.), Disziplinmanagement in der Schulklasse. Unterrichtsstörungen vorbeugen - Unterrichtsstörungen bewältigen 2. Aufl. Bern: Hans Huber.

Reiners, A. (1997). Praktische Erlebnispädagogik. Eine Sammlung motivierender Interaktionsspiele. Alling: Sandmann.

Rost, D. H. (2005). Interpretation und Bewertung pädagogisch-psychologischer Studien. Weinheim: Beltz.

Roth, G. (2011). Bildung braucht Persönlichkeit. Wie lernen gelingt. Stuttgart: Klett-Cotta.

Samdal, O., Nutbeam, D., Wold, B., \& Kannas, L. (1998). Achieving health and educational goals through school. A study of the importance of school climate and students' satisfaction with schools. Health Education Research, 13(3), 383-397.

Stanford, G. (2010). Gruppenentwicklung im Klassenraum und anderswo. Praktische Anleitung für Lehrer und Erzieher (8. Aufl.). Aachen: Hahn.

Steins, G., Bitau, K., \& Haep, A. (2014). Im Klassenzimmer. Sozialpsychologie des Schulalltags, Bd. II. Lengerich: Wolfgang Pabst Science Publishers. 
Zick, A. (2002). Wenn alle tatsächlich in einem Boot sitzen - Eine Studie zur Identifikation mit der Schule im Kontext einer erlebnispädagogischen Gruppenerfahrung.

Gruppendynamik und Organisationsberatung, 33, 213-228. 